CrossMark CrosSMark
click for updates

Cite this: J. Mater. Chem. B, 2015, 3, 3429

Received 19th January 2015, Accepted 23rd March 2015

DOI: 10.1039/c5tb00131e

www.rsc.org/MaterialsB

\section{Bigels formed via spinodal decomposition of unfolded protein}

\begin{abstract}
Alice Blumlein and Jennifer J. McManus*
Bigels (or double network gels) are an emerging class of tuneable soft materials characterized by two discrete but interpenetrating gel networks in which both networks contribute to the physical and mechanical properties of the material. We describe, for the first time, the formation of a bigel network from two different proteins. By careful control of solution conditions, kinetics and specific protein chemistry the inter-species interactions in the two protein system are weak compared with the intra-protein attraction, which leads to bigel formation. The resulting protein bigel has an elastic modulus four times greater than the combined elastic moduli of the parent gels, has an elastic response over several deformation cycles and is both thermo- and chemo-responsive. These gels have the potential to be used as biomimetics in tissue culture, in drug delivery or for biomedical applications such as wound healing.
\end{abstract}

\section{Introduction}

Hydrogels are widely used for a variety of pharmaceutical and medical applications, particularly tissue engineering, prosthetics, wound healing, ${ }^{1,2}$ and as environment-sensitive implantable delivery systems. ${ }^{1,3}$ Hydrogels are considered analogous to the extracellular matrix, providing a template for cell growth, a scaffold to induce cell differentiation and a conduit to deliver essential growth factors, making them ideal for use in cell culturing. ${ }^{4}$ Increasingly, medical applications require more sophisticated hydrogels with complexity beyond being inert and biologically compatible; ideally, they should also possess tuneable and bioactive characteristics. The development of such biomimetics is the focus of intense research. ${ }^{5,6}$

Bigels (or double networks) are an emerging class of tuneable soft materials characterized by two discrete but interpenetrating gel networks in which both networks contribute to the physical and mechanical properties of the material. ${ }^{7-10}$ Bigelation adds levels of complexity and functionality to hydrogels, since the underlying mechanical properties and topological features of the material may be tuned at nanometre and micrometer lengthscales, ${ }^{11}$ an important consideration given that biological processes are often sensitive to mechanical cues. ${ }^{12}$

Scaffolds for tissue engineering and drug delivery are often comprised of synthetic materials such as poly(glycolic acid), poly(lactic acid) and poly(acrylic acid). However, natural polymers, such as proteins are also ideal building blocks for biomedical hydrogels due to their inherent biocompatibility and biodegradability and that specific protein epitopes can be integrated for

Department of Chemistry, Maynooth University, Maynooth, Co. Kildare, Ireland. E-mail: jennifer.momanus@nuim.ie the controlled release of bioactive molecules. ${ }^{13,14}$ Fast-acting controlled release of drugs requires small thin hydrogels but traditional hydrogels lack mechanical stability and thus are not always suitable vectors. ${ }^{15}$ Indeed, tough hydrogels (highly stretchable with large fracture energies) are also required for strain bearing biomedical applications such replacement cartilage and bone grafting. ${ }^{8}$ For bigels, the mechanical properties of each component gel works synergistically to endow the gel with mechanical properties beyond those of the parent gels. Tough double network gels, composed of alginate and polyacrylamide and also of proteoglycans and glycosaminoglycans have been described recently, ${ }^{11}$ however, the development of a tough, proteinonly hydrogel would satisfy the mechanical and biocompatibility shortcomings of traditional hydrogels.

The term bigel has been used to describe several different types of bicontinuous and separate gelled networks such as an oleohydrogel formed from a mixture of a hydrogel and an organogel, ${ }^{16}$ a macroscopically heterogeneous construct comprised of two unique gel strips bonded together, ${ }^{17}$ and a binary interpenetrating colloidal gel. ${ }^{7,9,18}$ Furthermore, the term double network has also been used to describe gels composed of two gelled networks formed by either sequential covalent networking ${ }^{19}$ or by the formation of a combination of covalent and non-covalent networks. ${ }^{8,19}$ Phase-separated bicontinuous biopolymer materials have also been described. ${ }^{20-22}$

The materials described here were formed from two percolated, interpenetrating but discrete networks. The first network is formed by spinodal decomposition of thermally unfolded BSA and the second network is formed from gelatin which forms a percolated network of randomly organised linear filaments. ${ }^{23}$ The properties and microstructure of this bigel were measured using cavitation rheology, confocal microscopy and electron microscopy. 
The resulting protein bigel has an elastic modulus four times greater than the combined elastic moduli of the parent gels, has an elastic response over several deformation cycles and is both thermo- and chemo-responsive. These gels have the potential to be used as biomimetics, in tissue culture, in drug delivery or for biomedical applications such as wound healing. We also propose a mechanism that describes how the interspecies interactions in the two protein system are negligible compared with the intra-protein attraction for each species, which leads to bigel formation.

\section{Results and discussion}

Gels composed of BSA only (at low ionic strength) were prepared over a range of protein concentrations $\left(10-180 \mathrm{mg} \mathrm{ml}^{-1}\right.$; $\phi_{\mathrm{BSA}}=0.007$ to 0.133 ) and heated above the melt transition temperature $\left(T_{\mathrm{m}}\right)$ for BSA (determined using differential scanning calorimetry $^{24}$ ). BSA gels below a volume fraction $\phi_{\mathrm{BSA}}=0.059$ $\left(80 \mathrm{mg} \mathrm{ml}^{-1}\right.$ ) are transparent and very weak with elastic moduli in the region of $1 \mathrm{kPa}$. These gels were unstable and often collapsed. The elastic modulus was measured using cavitation rheology (CR), a technique that relates the pressure at which bubble formation in a soft material occurs $\left(P_{\mathrm{c}}\right)$ to Young's modulus $(E)$ :

$$
P_{\mathrm{c}}=\frac{5}{6} E+\frac{2 \gamma}{r}
$$

where $\gamma$ is the surface tension and $r$ is the diameter of the needle. ${ }^{25}$ At higher protein concentrations, ( $\left.90 \mathrm{mg} \mathrm{ml}^{-1} ; \phi_{\mathrm{BSA}}=0.066\right)$, more stable gels formed and the elastic modulus increased to $\sim 3.2 \mathrm{kPa}$
(Fig. 1, top right). As the concentration increased above $160 \mathrm{mg} \mathrm{ml}^{-1}$ $\left(\phi_{\mathrm{BSA}}=0.118\right)$ opaque gels formed (Fig. 1, top left). At volume fractions of $\phi_{\mathrm{BSA}}=0.133\left(180 \mathrm{mg} \mathrm{ml}^{-1}\right)$, gels with an elastic modulus of $52.2 \mathrm{kPa}$ were observed. Light microscopy of the turbid BSA gels revealed a percolated network of small monodisperse particles of $\sim 1 \mu \mathrm{m}$ in size.

CR can be used to assess if a reversible elastic deformation of the material occurs (cavitation) or if irreversible fracture is observed, by monitoring $P_{\mathrm{c}}$ following several pressurization and depressurization cycles. ${ }^{26}$ For $90 \mathrm{mg} \mathrm{ml}^{-1}$ BSA gels $\left(\phi_{\mathrm{BSA}}=0.066\right)$, partial reversibility of the cavitation process (60-70\%) was measured for larger needle radii during the second pressurisation cycle (Fig. 1, bottom left), although the $P_{\mathrm{c}}$ did decrease further with subsequent cycles. However, as the concentration of the BSA is increased resulting in gels with higher elastic modulus, irreversible fracture rather than cavitation is observed at $\phi_{\mathrm{BSA}}=0.111\left(150 \mathrm{mg} \mathrm{ml}^{-1}\right)$ and higher.

Gelatin is a well-studied material, although variability in the elastic modulus measured between different studies originates from variability in the gelatin itself, which is derived by acidic hydrolysis of collagen (type A) and type B, formed by basic hydrolysis. $^{27}$

Above a critical concentration of $30-40 \mathrm{mg} \mathrm{m}^{-1}$ (gelatin volume fraction, $\phi_{\mathrm{G}}=0.022$ to 0.029$)$ gelation was observed. Below this concentration, the solution remained liquid-like.

Transparent, gelatin only gels (at low ionic strength) were prepared over range of protein concentrations $\left(100-190 \mathrm{mg} \mathrm{ml}^{-1}\right.$, $\phi_{\mathrm{G}}=0.073$ to 0.139 ), resulting in materials with elastic modulii from 2.8 to $10.5 \mathrm{kPa}$ across this concentration range, which is consistent with previous work ${ }^{28}$ (Fig. 1, bottom right).
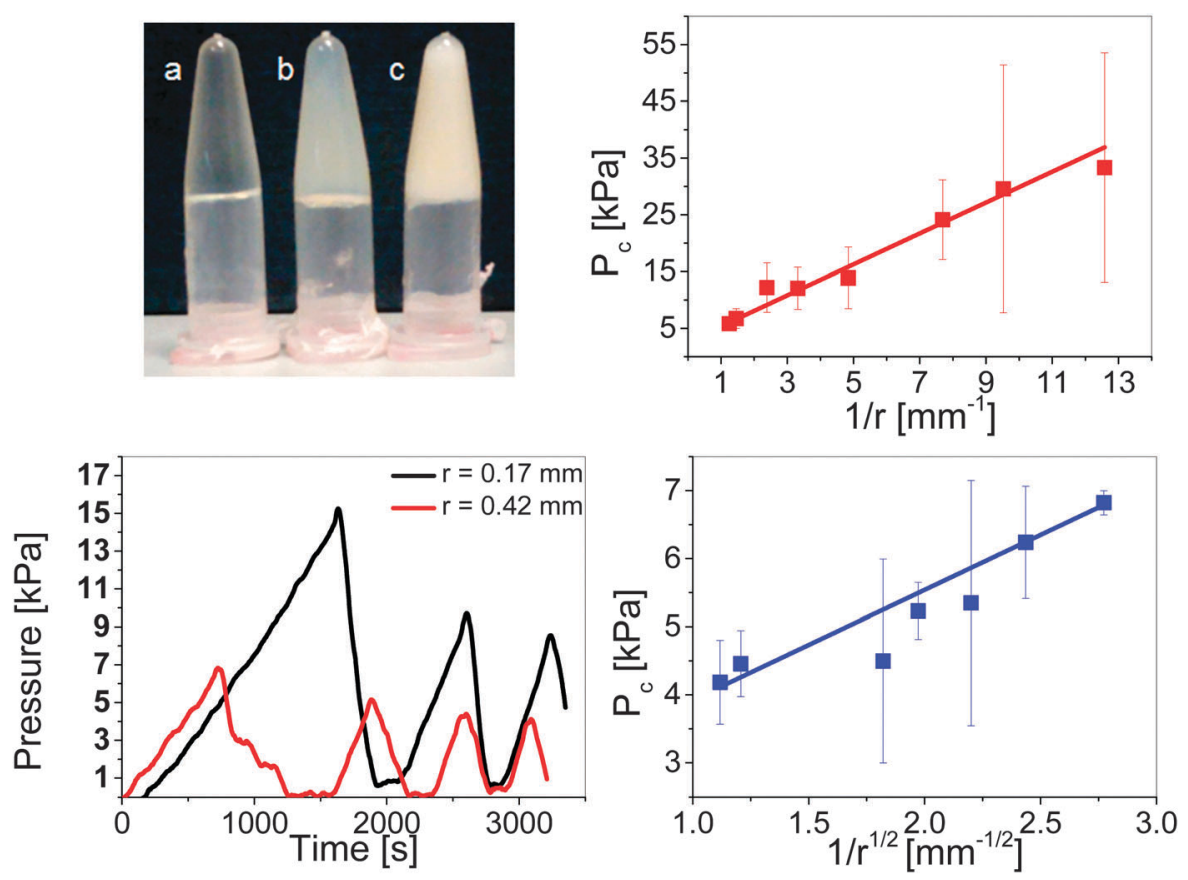

Fig. 1 (top left) BSA gels transition from being transparent at (a) $\phi_{\mathrm{BSA}}=0.066\left(90 \mathrm{mg} \mathrm{ml}^{-1}\right)$ to opaque at (b) $\phi_{\mathrm{BSA}}=0.118\left(160 \mathrm{mg} \mathrm{ml}^{-1}\right)$ and this opacity increases with increasing BSA concentration, (c) $\phi_{\mathrm{BSA}}=0.133\left(180 \mathrm{mg} \mathrm{ml}^{-1}\right)$. (top right) The elastic modulus of $\phi_{\mathrm{BSA}}=0.066\left(90 \mathrm{mg} \mathrm{ml}^{-1} \mathrm{BSA}\right)$ was found to be $3.2 \mathrm{kPa}$, using cavitation rheology. (bottom left) Cavitation rheology of $\phi_{\mathrm{BSA}}=0.066\left(90 \mathrm{mg} \mathrm{ml}^{-1}\right)$ BSA gels show the deformation process is partially (60-70\%) reversible. (bottom right) The elastic modulus of $\phi_{\mathrm{G}}=0.074$ (100 $\mathrm{mg} \mathrm{ml}^{-1}$ gelatin) was found to be $2.8 \mathrm{kPa}$ by CR. 
The deformation process for gelatin proceeds via irreversible fracture and the pressure at the onset of deformation has a $1 / r^{1 / 2}$ dependence (Fig. 1, bottom right), consistent with the scaling relationship observed during the fracture of polyacrylamide gels. ${ }^{6}$

BSA and gelatin in solution were initially mixed and then heated to temperatures above the $T_{\mathrm{m}}$ for the highest melting component, BSA in this case.

To form the bigel, solution conditions were selected such that gelation of both BSA and gelatin would occur independently, but in one pot, i.e. the net attraction between the two species was minimised so that each material had greater affinity itself than the other component, which is a requirement for true bigel formation. ${ }^{7,18}$

Initially, gels containing both protein components were formed at a gelatin volume fraction fixed at $\phi_{\mathrm{G}}=0.074\left(100 \mathrm{mg} \mathrm{ml}^{-1}\right)$ and BSA volume fractions varying between $\phi_{\mathrm{BSA}}=0.007$ to 0.074 (10-100 $\mathrm{mg} \mathrm{ml}^{-1}$ ). All gels were turbid. Light microscopy of the gels revealed clusters of spherical aggregates at lower BSA concentrations $\left(10 \mathrm{mg} \mathrm{ml}^{-1} ; \phi_{\mathrm{BSA}}=0.007\right)$ that transitioned to a percolated network at $40 \mathrm{mg} \mathrm{ml}^{-1}\left(\phi_{\mathrm{BSA}}=0.030\right)$ (Fig. 2, top). The most robust gels were formed at a 9:10 BSA/gelatin weight ratio $\left(\phi_{\text {Bigel }}=0.141\right)$; these gels were further probed to examine their mechanical properties and microstructure. The elastic modulus of the 9:10 protein bigel was $24.3 \mathrm{kPa}$, measured using CR (Fig. 2, middle). This is significantly higher than the elastic modulus for either parent gel and four times greater than the sum of the elastic moduli of each component gel at their initial concentration (Fig. 2, middle).

To probe the mechanical properties of the bigel further, we explored the reversibility of the deformation of the gel using CR. These measurements indicate that the deformation process is highly elastic. In fact, the critical pressure returns to $60-70 \%$ of the original value during the second and subsequent (up to 4) pressurisation cycles. This is somewhat surprising. We know from previous work and from our own CR measurements, that gelatin is subject to irreversible fracture after cavitation across the full range of $r$ values that we have probed. As such, a fracture event in the gelatin containing bigel may have resulted in a material which did not maintain its increased mechanical strength after a single deformation. Therefore, the protein bigel has both improved resistance to deformation (since the $P_{\mathrm{c}}$ is considerably higher than for a comparable BSA only gel) and superior retention of elasticity following multiple deformation cycles.

The microstructure of the gels was examined using Environmental Scanning Electron Microscopy (ESEM) and confocal microscopy. The ESEM images indicate that the gel surface consists of a mono-disperse percolated network of spherical structures of $\sim 1 \mu \mathrm{m}$ in diameter, consistent with the light microscopy of the BSA only gels (Fig. 3, left). Confocal microscopy reveals that each protein component forms an independent network (Fig. 3, right). The BSA gel is a branched structure of connected spherical aggregates, consistent with a network formed as a result of spinodal decomposition. ${ }^{29}$

As expected, no distinct structural features for the gelatin gel were observed. When the images were superimposed
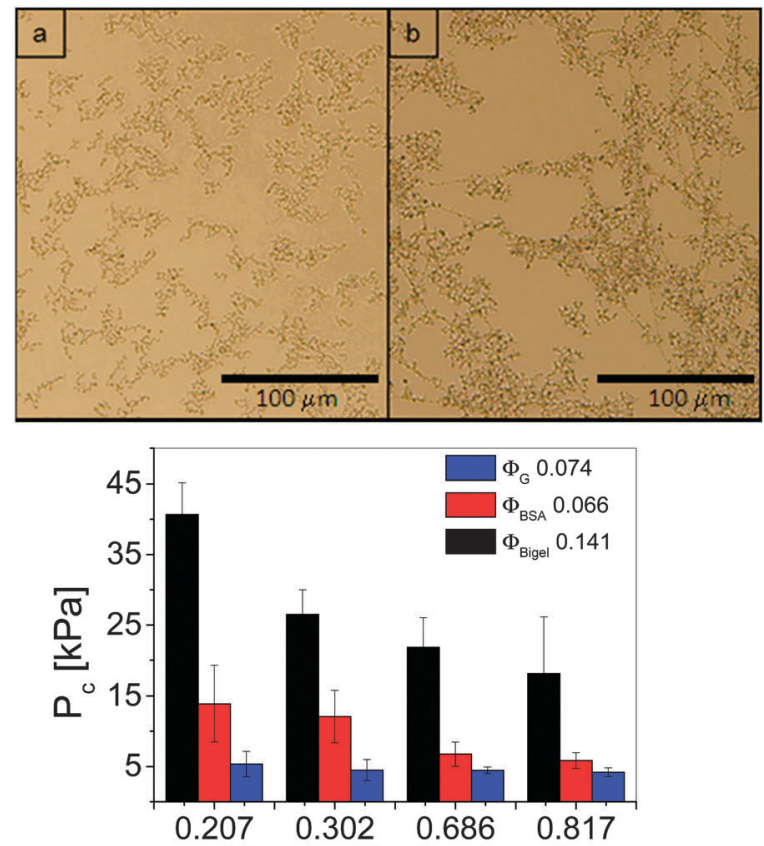

Needle Radius [mm]

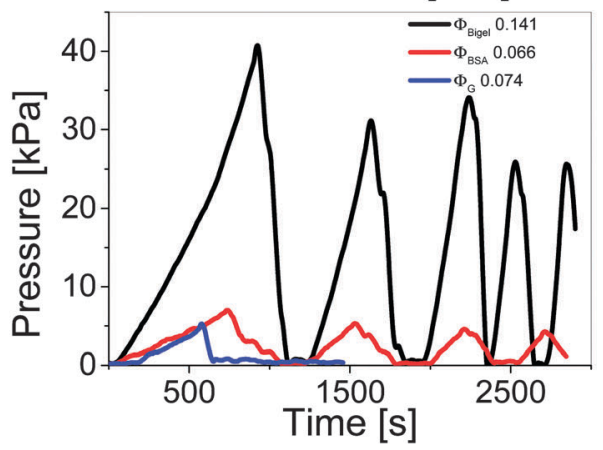

Fig. 2 (top) Light microscopy image of gel formed from (a) $\phi_{\mathrm{BSA}}=0.0074$ (10 $\left.\mathrm{mg} \mathrm{ml}^{-1} \mathrm{BSA}\right)$ (b) $\phi_{\mathrm{BSA}}=0.0292\left(40 \mathrm{mg} \mathrm{ml}^{-1}\right)$ BSA showing the transition from clusters to a percolated network of spherical aggregates. (middle) Comparison of elastic moduli and the critical pressure for $\phi_{\mathrm{G}}=0.007$ (10\% gelatin), $\phi_{\mathrm{BSA}}=0.066(9 \% \mathrm{BSA})$ and $\phi_{\text {Bigel }}=0.141$ (9:10 BSA : gelatin) for different needle radii. (bottom) Cavitation rheology of bigel show the deformation process is partially (60-70\%) reversible.

(Fig. 3, far right), a discrete spatial distribution of each gel component is observed, which together form an interpenetrating bigel network. At a fracture point in the gel, it is clear that only the gelatin component of the gel is fractured irreversibly. The structure of the BSA particle gel remains, bridging the gap between the fractured portions of the bigel. Hence, there may be a mechanism by which the new bigel material retains its elasticity after deformation.

The new bigel material has an elastic modulus four times greater than the sum of the elastic moduli of the parent gels from which it was derived. In the bigel, after an initial deformation, which irreversibly fractures the gelatin component, a BSA particle gel structure remains with an estimated elastic modulus of $\sim 17 \mathrm{kPa}$, considerably greater than would be expected $(\sim 3 \mathrm{kPa})$ for the single component gel at the same BSA concentration. 

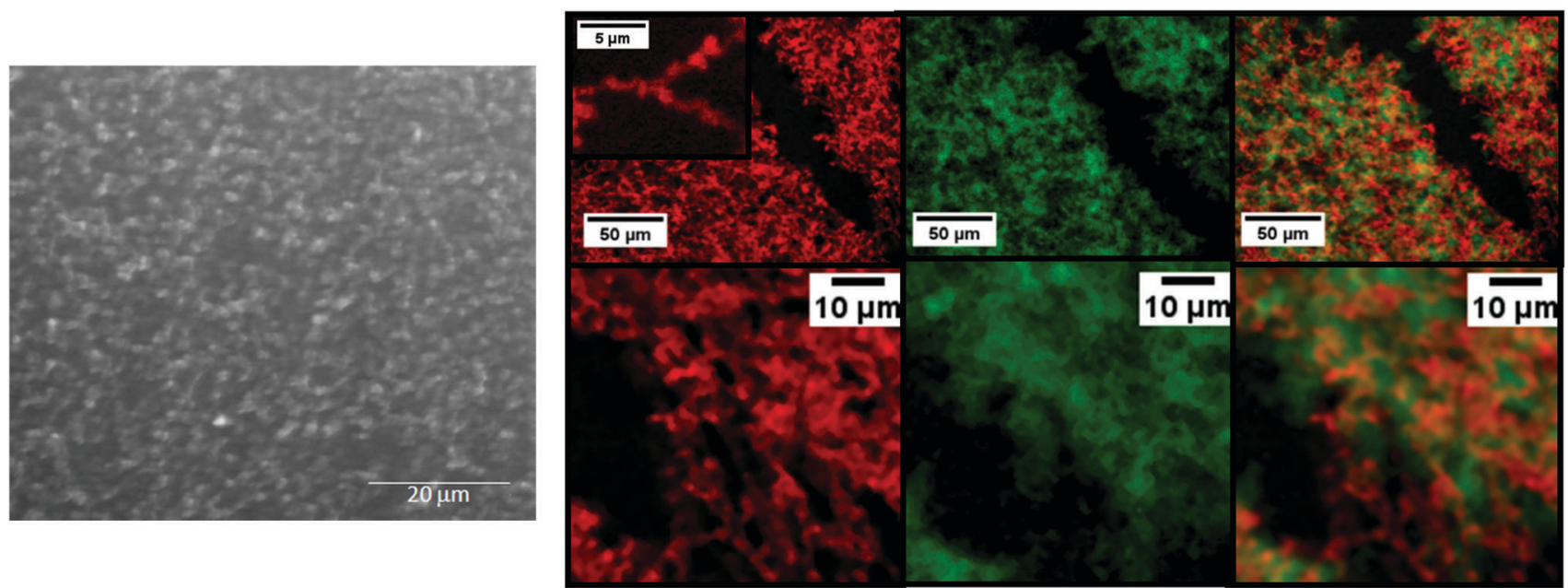

Fig. 3 (left) Environmental scanning electron micrograph of gel formed from $\phi_{\text {Bigel }}=0.141\left[100 \mathrm{mg} \mathrm{ml}^{-1}\right.$ gelatin $\left(\phi_{\mathrm{G}}=0.074\right)$ and $90 \mathrm{mg} \mathrm{ml} \mathrm{l}^{-1} \mathrm{BSA}\left(\phi_{\mathrm{BSA}}\right.$ $=0.066)$, showing a surface composed of $1-2 \mu \mathrm{m}$ spheres. (right) Confocal micrograph for the bigel $\phi_{\text {Bigel }}=0.141\left[100 \mathrm{mg} \mathrm{ml}^{-1} \mathrm{gelatin}\left(\phi_{\mathrm{G}}=0.074\right)\right.$ and $\left.90 \mathrm{mg} \mathrm{ml}^{-1} \mathrm{BSA}\left(\phi_{\mathrm{BSA}}=0.066\right)\right]$. Gelatin labelled with FITC appears green, BSA labelled with Dylight 633 appears red. The overlay of the two images indicates that the gel networks are discrete but interpenetrating (far right panel).

Numerical simulations for a bigel network formed from colloidal particles functionalised by single stranded DNA has indicated that bigels can tolerate increased stress compared to single one-component gels. ${ }^{9}$ For our protein bigels the additional mechanical strength and elasticity appear to arise from a difference in the way the BSA particle gel behaves in the presence of gelatin. To identify the source from which the additional mechanical strength is derived, we prepared two BSA gels at the same total volume fraction as the BSA/gelatin bigel, $(\phi=0.141)$. The first was a mixture of BSA and PEG and the second was BSA only gel $\left(\phi_{\mathrm{BSA}}=0.141\right)$. The BSA-PEG gels were transparent and there was no significant increase in the elastic modulus compared to the BSA only gel. Therefore the increase in mechanical strength of the BSA/gelatin bigel is not simply due macromolecular crowding as a result of a higher total volume fraction. For the BSA only gel at a total volume fraction of $\phi_{\mathrm{BSA}}=0.141$, the elastic modulus is considerably higher than for the bigel at the same volume fraction. However, it is not elastic and fractures irreversibly upon deformation.

A remarkable thermo-responsiveness of the bigels was also observed. At $4{ }^{\circ} \mathrm{C}$ and at room temperature, the gels swell in the presence of excess liquid (Fig. 4, top). However, at $37^{\circ} \mathrm{C}$, there is a dramatic transition to shrinking behavior. The potential for use in drug delivery applications is therefore clear. Using FITC (in solution) as a model, we see that the release is best at $37^{\circ} \mathrm{C}$, which is consistent with the shrinking behavior of the gel at this temperature. The release is sustained over at least a 48 hour period.

For true bigelation to occur, it must be clear how the inter-species attraction is negligible compared with the intra-molecular interactions for each species. For the first bigels described experimentally, this was elegantly achieved by functionalisation of colloidal particles with single stranded DNA. ${ }^{7}$

When using proteins as starting materials, careful consideration must be given to the mechanism of bigel formation to

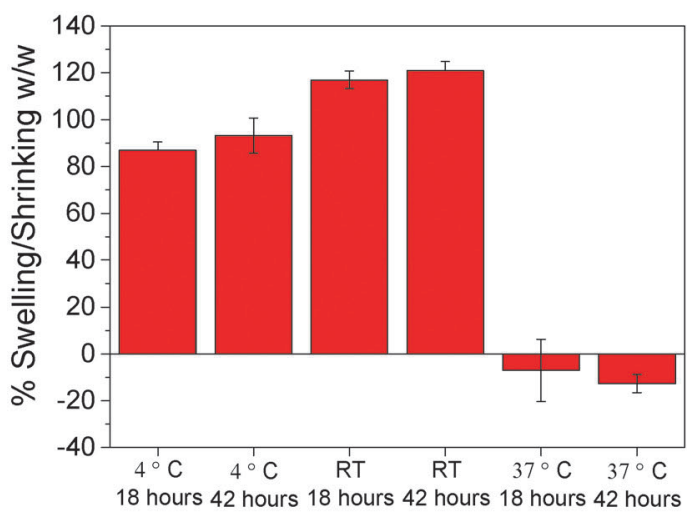

Solution Conditions

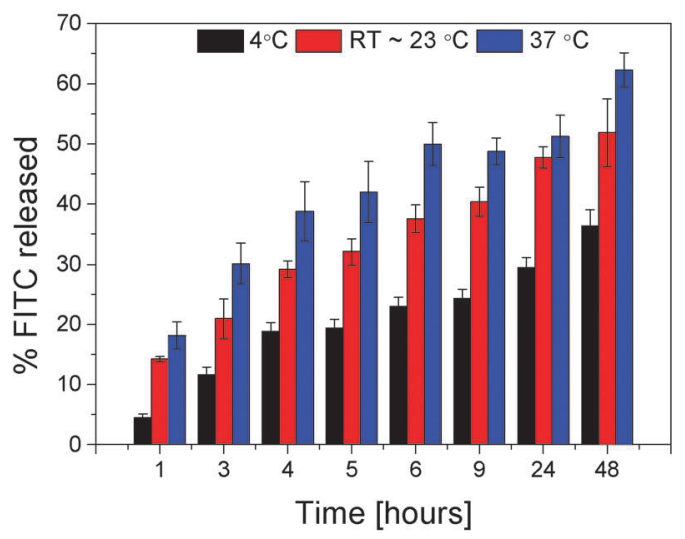

Fig. 4 (top) Swelling/shrinking behaviour of bigel in response to heat treatment. (bottom) FITC release profile for the 9:10 BSA/gelatin bigel indicating improved release of FITC at physiological temperature.

ensure that this condition is met. In this case, we have controlled solution conditions, kinetics and the specific chemistry of each component to achieve bigelation. Both protein solutions are prepared close to the isoelectric point of gelatin, resulting in a 
net charge close to zero for the gelatin component. For BSA however $(\mathrm{pI}=4.7)$, some residual charge remains, which at the low concentrations of protein in the initial mixture, prevents protein aggregation from occurring. Once heated to $90{ }^{\circ} \mathrm{C}$, we abruptly unfold BSA to deep within the spinodal region of the phase diagram, forming the arrested percolated network that we observe in both the BSA only gels at $\phi_{\mathrm{BSA}}=0.066$ and for the bigels at a minimum $\phi_{\text {Bigel }}=0.104$. The remarkable homogeneity of the particle sizes indicates that in fact spinodal decomposition occurs. The question is then why gelatin, which is in a molten globular state under these conditions ${ }^{30}$ does not associate with the BSA at this point. It is well known that unfolded BSA forms irreversible protein aggregates via covalent inter-protein disulfide bonds. ${ }^{31}$ We believe that spinodal decomposition is followed by aggregate formation within the arrested structure. Opacity in the bigel occurs at $90{ }^{\circ} \mathrm{C}$, before the gelatin network forms. Since gelatin type A has no cysteine residues, it will not covalently attach to the BSA network. Furthermore, gelatin is predominantly hydrophilic, both above and below its melt transition temperature, and hence interspecies attraction mediated by hydrophobic amino acid residues will be minimised. ${ }^{32}$ There is therefore a clear pathway for bigel formation to occur. It is important to note, that spinodal decomposition must precede aggregation, to prevent nucleation driven amorphous aggregate formation.

Once arrest and aggregation within the BSA gel is complete, gelation of gelatin occurs around the pre-existing BSA network upon cooling resulting in two discrete but interpenetrating
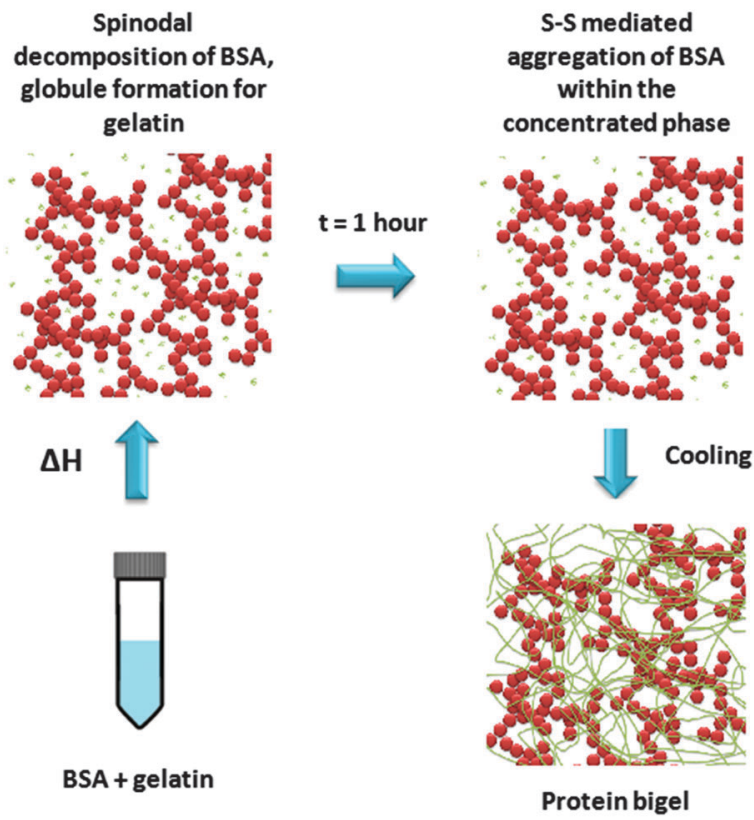

Fig. 5 Proposed mechanism for protein bigel formation. Beginning at step 1 (bottom left), the two component proteins are mixed and BSA gel formation is achieved by spinodal decomposition of the unfolded protein above its melt transition temperature, $T_{\mathrm{m}}$. Irreversible aggregation mediated by intermolecular disulfide bridges within the arrested structure stabilises the BSA gel. Gelatin formation around the pre-existing BSA gel occurs upon re-cooling. gels. This is consistent with the experimental evidence. This proposed mechanism for bigel formation is illustrated in Fig. 5.

Furthermore, it is significant that the protein bigels that form here have the same scaling in mechanical behaviour as the previously described DNA bigels. ${ }^{10}$ The preservation of the particle network structure in both the BSA subgel and the BSA/ gelatin bigel also indicates that there is no gelatin association during bigel formation. Indeed, the density at which bigel formation occurs $\left(\phi_{\text {Bigel }}=0.141\right)$, and composition of the material $\left(\phi_{\mathrm{BSA}} / \phi_{\text {Bigel }}=0.47\right)$, are within the region of the phase diagram in which two dynamically arrested interpenetrating gels are predicted to form. ${ }^{7}$

\section{Experimental}

Gelatin (from bovine skin) and polyethylene glycol 20000 were purchased from Sigma-Aldrich (St. Louis, MO, USA) and bovine serum albumin was purchased from Fisher scientific. Solutions of hydrochloric acid, calcium chloride and sodium chloride were prepared using analytical grade reagents. Fluorescein isothiocyanate (FITC) and Dylight 633 were purchased from Thermo scientific. All reagents were used without further purification.

For all experiments (with the exception of confocal microscopy) BSA and gelatin samples were prepared at concentrations between $10 \mathrm{mg} \mathrm{ml}^{-1}$ and $200 \mathrm{mg} \mathrm{ml}^{-1}\left(\phi_{\mathrm{BSA}}=0.007\right.$ to 0.148). Gelatin samples were heated at $80{ }^{\circ} \mathrm{C}$ for 10 minutes in a water bath (to ensure that the gelatin was fully dissolved). BSA and gelatin solutions at appropriate concentrations were mixed at room temperature to prepare bigels. For BSA, differential scanning calorimetry was used to determine the melt transition temperature, $T_{\mathrm{m}}$, and to quantify the degree of refolding to the native conformation following thermal denaturation. ${ }^{24}$ Samples were then heated at $80{ }^{\circ} \mathrm{C}$ for 1 hour in a water bath and cooled at room temperature for a further 1 hour. Since BSA and gelatin gels are known to cure (form additional bonds over time), it was necessary to ensure that the gels were aged in the same fashion. ${ }^{33}$ The gels containing PEG were prepared using the same procedure. For swelling/shrinking and controlled release experiments, the gels were cut into disks of approximately $7 \times 3 \mathrm{~mm}$ and were incubated in $5 \mathrm{ml}$ of buffer at the specified temperature. For the controlled release experiments, FITC was added at a final concentration of $0.03 \mu \mathrm{g} \mathrm{ml} \mathrm{m}^{-1}$ to samples prior to heating.

Light microscopy was performed on an Olympus BX61 microscope, equipped with a digital imaging system was used to examine the gels at $100 \times$ magnification. Cell $^{\mathrm{F}}$ software was used to view and record the images and all image analysis was done using ImageJ software. ${ }^{34}$

Environmental scanning electron microscopy (ESEM) images were obtained for the $90 \mathrm{mg} \mathrm{ml}^{-1} \mathrm{BSA}\left(\phi_{\mathrm{BSA}}=0.066\right)$ and $100 \mathrm{mg} \mathrm{ml}^{-1}$ gelatin $\left(\phi_{\mathrm{G}}=0.074\right)$ gels, and for the 9:10 bigel $\left(\phi_{\text {Bigel }}=0.141\right)$. The images were obtained using a FEI Quanta 3D FEG DualBeam (FEI Ltd, Hillsboro, USA) at the Nano Imaging and Material Analysis Centre, University College Dublin, Ireland. 
Confocal microscopy images were obtained for bigels $\left(\phi_{\text {Bigel }}=\right.$ 0.141 ) formed from $90 \mathrm{mg} \mathrm{ml}^{-1} \mathrm{BSA}\left(\phi_{\mathrm{BSA}}=0.066\right)$ and $100 \mathrm{mg} \mathrm{ml}^{-1}$ gelatin $\left(\phi_{\mathrm{G}}=0.074\right)$, using a Zeiss LSM 710 NLO at the Royal College of Surgeons, Dublin, Ireland. FITC was used to fluorescently label the gelatin (the protein appears green), and, DyLight 633 was used to fluorescently label the BSA (the protein appears red). Labelling was performed as per the suppliers instructions. Each labelled protein was repeatedly washed by ultrafiltration for 5 minutes, using Amicon Ultra-4 MWCO $10 \mathrm{kDa}$ centrifugal devices at $4000 \times g$, until no unbound fluorophore was detected in the filtrate. A final spin at $7000 \times g$ for 10 minutes was used to concentrate the sample before preparing bigels as indicated above.

Cavitation rheology (CR) has been shown to measure the Young's modulus of soft materials, including polyacrylamide gels, the eye lens and vitreous. ${ }^{25,26,35}$ The cavitation rheology instrument was built in-house and comprises of a syringe pump (New Era) regulating a syringe (Hamilton GASTIGHT ${ }^{\circledR}$ ), connected to an pressure sensor (Omega Engineering) and blunt end needles (Fisher scientific). The syringe pump was controlled from a personal computer using a custom written programme using LabVIEW software (National Instruments), which also recorded pressure at the needle tip during the experiments. During cavitation rheology a syringe is inserted into the sample and a cavity is formed at the tip by increasing the pressure of the medium within the syringe. The sample was placed on a translation stage, which was adjusted until the position of the needle was at the centre of the sample. Compression of the medium within the syringe was then induced a rate of $400 \mu \mathrm{l} \mathrm{min}{ }^{-1}$ when air was used as the cavitation medium and $50 \mu \mathrm{min}^{-1}$ when water was used for cavitation. At a given pressure, the critical pressure $\left(C_{\mathrm{p}}\right)$ the gel began to deform rapidly, such a deformation may be elastic, resulting in the formation of a cavity or inelastic which leads to a permanent fracture. The reversibility of these deformations was tested by running the pump for a series of pressurization and depressurisation cycles. Reversible cavitation was determined to occur when a critical pressure was maintained for multiple deformation events. Where the deformation proceeded by cavitation, $C_{\mathrm{p}}$ scales linearly with $1 / r$ (the internal radius of the needle); whereas $C_{\mathrm{p}}$ scales with $1 / r^{1 / 2}$ when a fracturing event occurred. ${ }^{26}$

\section{Conclusions}

A bigel network, composed of a percolated particle gel formed by spinodal decomposition of unfolded BSA and a discrete but interpenetrating network of gelatin has been formed. The bigel has significantly enhanced mechanical strength compared with either individual component, which is derived from the synergistic interactions between the networks, ${ }^{9,10}$ resulting in a biocompatible material with physical properties that give it the potential to be used as a drug delivery material or as a support for cell growth. It is mechanically elastic after repeated deformation cycles and was shown to release FITC at $37{ }^{\circ} \mathrm{C}$ over a sustained period.

The formation of this two-protein bigel is achieved by selecting components for which both solution conditions and kinetics can be tuned to ensure minimal interactions between the two species. Once heated above it's melt transition temperature, BSA rapidly unfolds within the spinodal region of the phase diagram. This is followed by irreversible aggregation of BSA, via $\mathrm{S}-\mathrm{S}$ bond formation within the arrested phase. Gelatin remains in a molten globule state until the solution is cooled, and then forms a gel network around the pre-existing BSA network, resulting in the formation of two discrete, but interpenetrating gels, a bigel.

Both the CR measurements and the confocal microscopy images provide consistent evidence to support the theory that the mechanism of energy dissipation in the bigel originates within the BSA component of the gel and that this would account for the remarkable rheological properties of this double network bigel.

\section{Acknowledgements}

This work was made possible by financial support from the Maynooth University Teaching Fellowship and the Science Foundation Ireland Stokes Lectureship to JJMcM. We thank Alfred Crosby and Jun Cui for their advice on building the CR instrument. We thank Noel Williams and Justine O'Sullivan for their assistance in building the CR instrument. We thank Brenton Cavanagh for his assistance with the confocal microscopy.

\section{References}

1 Q. Q. Dou, S. S. Liow, E. Ye, R. Lakshminarayanan and X. J. Loh, Adv. Healthcare Mater., 2014, 3, 977-988.

2 A. Lisman, B. Butruk, I. Wasiak and T. Ciach, J. Biomater. Appl., 2014, 28, 1386-1396.

3 Y. Zhang, H. F. Chan and K. W. Leong, Adv. Drug Delivery Rev., 2013, 65, 104-120.

4 S. Van Vlierberghe, P. Dubruel and E. Schacht, Biomacromolecules, 2011, 12, 1387-1408.

5 M. Verhulsel, M. Vignes, S. Descroix, L. Malaquin, D. M. Vignjevic and J.-L. Viovy, Biomaterials, 2014, 35, 1816-1832.

6 M. Lavine, M. Frisk and E. Pennisi, Science, 2012, 338, 899.

7 F. Varrato, L. Di Michele, M. Belushkin, N. Dorsaz, S. H. Nathan, E. Eiser and G. Foffi, Proc. Natl. Acad. Sci. U. S. A., 2012, 109, 19155-19160.

8 J.-Y. Sun, X. Zhao, W. R. K. Illeperuma, O. Chaudhuri, K. H. Oh, D. J. Mooney, J. J. Vlassak and Z. Suo, Nature, 2012, 489, 133-136.

9 L. Di Michele, F. Varrato, J. Kotar, S. H. Nathan, G. Foffi and E. Eiser, Nat. Commun., 2013, 4, 2007.

10 L. Di Michele, D. Fiocco, F. Varrato, S. Sastry, E. Eiser and G. Foffi, Soft Matter, 2014, 10, 3633-3648.

11 X. Zhao, Soft Matter, 2014, 10, 672-687.

12 M. J. Buehler and T. Ackbarow, Comput. Meth. Biomech. Biomed. Eng., 2008, 11, 595-607.

13 J. Zhu and R. E. Marchant, Expert Rev. Med. Devices, 2011, 8, 607-626. 
14 X. Zhang, H. Zhou, Y. Xie, C. Ren, D. Ding, J. Long and Z. Yang, Adv. Healthcare Mater., 2014, 3, 1804-1811.

15 Y. Qiu and K. Park, Adv. Drug Delivery Rev., 2001, 53, 321-339.

16 M. M. Ibrahim, S. A. Hafez and M. M. Mahdy, Asian J. Pharm. Sci., 2013, 8, 48-57.

17 J. Zhang, J. Wu, J. Sun and Q. Zhou, Soft Matter, 2012, 8, 5750-5752.

18 A. Goyal, C. K. Hall and O. D. Velev, J. Chem. Phys., 2010, 133, 064511.

19 J. P. Gong, Y. Katsuyama, T. Kurokawa and Y. Osada, Adv. Mater., 2003, 15, 1155-1158.

20 J. R. Stokes, B. Wolf and W. J. Frith, J. Rheol., 2001, 45, 1173-1191.

21 S. Wassen, R. Bordes, T. Geback, D. Bernin, E. Schuster, N. Loren and A. M. Hermansson, Soft Matter, 2014, 10, 8276-8287.

22 S. Kasapis, Crit. Rev. Food Sci. Nutr., 2008, 48, 341-359.

23 M. Djabourov, Contemp. Phys., 1988, 29, 273-297.

24 A. Blumlein and J. J. McManus, BBA, Biochim. Biophys. Acta, Proteins Proteomics Proteins Proteomics, 2013, 1834, 2064-2070.
25 J. A. Zimberlin, N. Sanabria-DeLong, G. N. Tew and A. J. Crosby, Soft Matter, 2007, 3, 763-767.

26 S. Kundu and A. J. Crosby, Soft Matter, 2009, 5, 3963-3968. 27 M. Djabourov, J. Leblond and P. Papon, J. Phys., 1988, 49, 319-332.

28 J. L. Kavanagh, T. Menand and K. A. Daniels, Tectonophysics, 2013, 582, 101-111.

29 D. Gebauer, M. Kellermeier, J. D. Gale, L. Bergstrom and H. Colfen, Chem. Soc. Rev., 2014, 43, 2348-2371.

30 J. L. Gornall and E. M. Terentjev, Soft Matter, 2008, 4, 544-549.

31 T. Considine, H. A. Patel, S. G. Anema, H. Singh and L. K. Creamer, Innovative Food Sci. Emerging Technol., 2007, 8, 1-23.

32 A. Bhattacharjee and M. Bansal, IUBMB Life, 2005, 57, 161-172.

33 S. M. Tosh, A. G. Marangoni, F. Ross Hallett and I. J. Britt, Food Hydrocolloids, 2003, 17, 503-513.

34 C. A. Schneider, W. S. Rasband and K. W. Eliceiri, Nat. Methods, 2012, 9, 671-675.

35 J. Cui, C. Hee Lee, A. Delbos, J. J. McManus and A. J. Crosby, Soft Matter, 2010, 6, 3632-3635. 